\title{
The Influence of LOS Components on the Statistical Properties of the Capacity of Amplify-and-Forward Channels
}

\author{
Gulzaib RAFIQ, Matthias PÄTZOLD \\ Faculty of Engineering and Science, University of Agder, Grimstad, Norway \\ E-mail: \{gulzaib.rafiq, matthias.paetzold\}@uia.no \\ Received February 26, 2009; revised March 5, 2009; accepted March 7, 2009
}

\begin{abstract}
Amplify-and-forward channels in cooperative networks provide a promising improvement in the network coverage and system throughput. Under line-of-sight (LOS) propagation conditions in such cooperative networks, the overall fading channel can be modeled by a double Rice process. In this article, we have studied the statistical properties of the capacity of double Rice fading channels. We have derived the analytical expressions for the probability density function (PDF), cumulative distribution function (CDF), levelcrossing rate (LCR), and average duration of fades (ADF) of the channel capacity. The obtained results are studied for different values of the amplitudes of the LOS components in the two links of double Rice fading channels. It has been observed that the statistics of the capacity of double Rice fading channels are quite different from those of double Rayleigh and classical Rice fading channels. Moreover, the presence of an LOS component in any of the two links increases the mean channel capacity and the LCR of the channel capacity. The validity of the theoretical results is confirmed by simulations. The results presented in this article can be very useful for communication system designers to optimize the performance of cooperative networks in wireless communication systems.
\end{abstract}

Keywords: Amplify-and-Forward Channels, Channel Capacity, Cooperative Networks, Line-of-Sight Component, Double Rice Process, Double Rayleigh Process, Level-Crossing Rate, Average Duration of Fades

\section{Introduction}

Increased network coverage, improved link quality, and provision of new applications with increased mobility support are the basic demands imposed on future wireless communication systems. One promising solution to fullfil these requirements is the use of cooperative diversity techniques [1-3]. Single-antenna mobile stations in cooperative networks assist each other to relay the transmitted signal from the source mobile station (SMS) to the destination mobile station (DMS). The cooperation of single-antenna mobile stations in such networks to share their antennas for transmission of the signal makes it possible to form the so-called virtual multiple-input multiple-output (MIMO) system [4], thus, achieving the diversity gain. Moreover, such cooperation between mobile stations results in increased network coverage with

February 26, 2009. The material in this paper is based on "On the Statistical Properties of the Capacity of Amplify-and-Forward Channels Under LOS Conditions", by Gulzaib Rafiq and Matthias Pätzold which appeared in the proceedings of 11th IEEE International Conference on Communications Systems, ICCS 2008, Guangzhou, China, November 2008. C2007 IEEE. enhanced mobility support.

For the development and analysis of wireless communication systems that exploit cooperative diversity, a solid knowledge of the multipath fading channel characteristics is required. Recent studies illustrate that mobile-to-mobile (M2M) fading channels associated with relay-based cooperative networks under non-line-of-sight (NLOS) propagation conditions in different propagation scenarios can be modeled either as a double Rayleigh process [5-8] or an NLOS second-order scattering (NLSS) process [9]. On the other hand, different scenarios under LOS propagation conditions lead to modeling the overall M2M fading channel either by a double Rice process [10], a single-LOS double-scattering (SLDS) process [11], a single-LOS second-order scattering (SLSS) process [9], or a multiple-LOS second-order scattering (MLSS) process [12,13]. These studies provide results for the statistical characterization of M2M fading channels in cooperative networks under different propagation conditions. The impact of double Rayleigh fading on the performance of a communication system is investigated in [14]. Even with all this research going on, 
the important question regarding the maximum possible information transfer rate in such fading channels is still unanswered. Thus, the purpose of this paper is to fill in this gap in information regarding the capacity of amplify-and -forward channels in cooperative networks.

Studies pertaining to the analysis of the outage capacity of double Rayleigh channels can be found in $[15,16]$. However, to the best of the authors' knowledge, the statistical properties of the capacity of double Rice channels have never been investigated. The analysis of the statistical properties of the channel capacity can be very helpful to study the dynamic behavior of the channel capacity. Here, the statistical properties of interest include the PDF, CDF, LCR, and ADF of the channel capacity. The PDF and CDF of the channel capacity provide the information regarding mean value and variance of the channel capacity. The LCR and ADF of the channel capacity, on the other hand, give a deep insight into the temporal variations of the channel capacity [17]. The LCR of the channel capacity is a measure of the rate at which the channel capacity crosses a certain threshold level from up to down or vice versa. However, the ADF of the channel capacity is defined as the average duration of time over which the channel capacity is below a certain threshold level $[18,19]$.

In this paper, we have investigated the statistical properties of the capacity of amplify-and-forward channels in cooperative networks. The transmitted signal from the SMS is received at the DMS via a mobile relay (MR). The MR amplifies the received signal and forwards it to the DMS. We have assumed that there is no direct transmission link between the SMS and the DMS. Moreover, it is also assumed that the LOS components exist in both of the transmission links, i.e., the SMS-MR and MRDMS links. Hence, the overall fading channel is modeled by a double Rice process [10]. We have derived exact analytical expressions for the PDF, CDF, LCR, and ADF of the channel capacity of double Rice channels. The results are studied for different values of the amplitudes of the LOS components in the two transmission links of double Rice channels. It has been observed that the statistics of the capacity of double Rice channels are quite different from those of double Rayleigh and classical Rice/Rayleigh channels. Specifically, for medium and high signal levels, the presence of LOS components in the two cascaded transmission links increases the mean channel capacity and the LCR of the channel capacity. However, it results in a decrease in the ADF of the channel capacity.

The rest of the paper is organized as follows. In Section 2, we describe briefly the double Rice channel model and some of its statistical properties. The statistical properties of the capacity of double Rice channels are studied in Section 3. Section 4 presents the statistical properties of the capacity of double Rayleigh channels. Numerical results are discussed in Section 5. Finally, the concluding remarks are given in Section 6.

\section{The Double Rice Channel Model}

In cooperative networks employing amplify-and-forward relay, the channel between the SMS and the DMS via a MR can be represented as a concatenation of the SMSMR and MR-DMS channels [8,10]. Figure 1 depicts an example of the transmission link from the SMS to the DMS via the MR in such amplify-and-forward relaybased networks. For the case when an LOS component is present in both of the transmission links, i.e., the SMSMR link and the MR-DMS link, the overall fading channel can be modeled as a product of two non-zero- mean complex Gaussian processes given by [10].

$$
\chi(t)=A_{\mathrm{MR}} \mu_{\rho}^{(2)}(t) \mu_{\rho}^{(1)}(t)
$$

where $A_{\mathrm{MR}}$ is a real positive constant representing the relay gain and $\mu_{\rho}^{(i)}(t)=\mu^{(i)}(t)+m^{(i)}(t) \quad(i=1,2)$ models the fading in the $i$ th link. Here, $\mu^{(i)}(t)$ denotes the scattered component and $m^{(i)}(t)$ is the LOS component. The scattered component $\mu^{(i)}(t)$ can be modeled in the complex baseband as a complex Gaussian process with zero mean and variance $2 \sigma_{i}^{2}$, i.e., $\mu^{(i)}(t)=\mu_{1}^{(i)}(t)+j \mu_{2}^{(i)}(t) \quad$ where, $\mu_{1}^{(i)}(t)$ and $\mu_{2}^{(i)}(t)$ are the underlying zero-mean real-valued Gaussian processes. The LOS component $m^{(i)}(t)$ having amplitude $\rho_{i}$, Doppler frequency $f_{\rho_{i}}$, and phase $\theta_{\rho_{i}}$ i can be expressed as $m^{(i)}(t)=\rho_{i} e^{j\left(2 \pi f_{\rho i} t+\theta_{\rho i}\right)}$. Let $f_{\rho_{\mathrm{SMS}}}$, $f_{\rho_{\mathrm{MR}}}$, and $f_{\rho_{\mathrm{DMS}}}$ represent the respective Doppler frequencies of the SMS, MR, and DMS, then it can be easily observed from Figure 1 that $f_{\rho_{1}}=f_{\rho_{\mathrm{SMS}}}+f_{\rho_{\mathrm{MR}}}$ and $f_{\rho_{2}}=f_{\rho_{\mathrm{MR}}}+f_{\rho_{\mathrm{DMS}}}$. The envelope of the process $\chi(t)$ in (1) results in a double Rice process given by [10]

$$
\begin{aligned}
\Xi(t) & =|\chi(t)|=\left|A_{\mathrm{MR}} \mu_{\rho}^{(1)}(t) \mu_{\rho}^{(2)}(t)\right| \\
& =A_{\mathrm{MR}} \xi_{1}(t) \xi_{2}(t)
\end{aligned}
$$

where $\xi_{i}(t)(i=1,2)$ represents the classical Rice process.

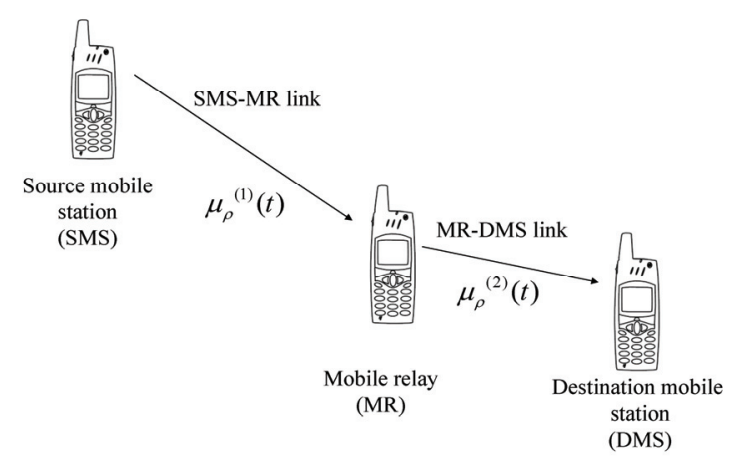

Figure 1. The propagation scenario describing double Rice fading channels. 
The PDF of double Rice processes $\Xi(t)$ is given by [10]

$$
\begin{aligned}
p_{\Xi}(z)= & \frac{z}{\sigma_{1}^{2} \sigma_{\mathrm{MR}}^{2}} \int_{0}^{\infty} \frac{1}{y} e^{-\frac{(z / y)^{2}+\rho_{1}^{2}}{2 \sigma_{1}^{2}}} e^{-\frac{y^{2}+\rho_{\mathrm{MR}}^{2}}{2 \sigma_{\mathrm{MR}}^{2}}} \\
& I_{0}\left(\frac{z \rho_{1}}{y \sigma_{1}^{2}}\right) I_{0}\left(\frac{y \rho_{\mathrm{MR}}}{\sigma_{\mathrm{MR}}^{2}}\right) d y, \quad z \geq 0
\end{aligned}
$$

where $I_{0}(\cdot)$ is the modified Bessel function of the first kind of order zero [20], $\sigma_{\mathrm{MR}}^{2}=\left(A_{\mathrm{MR}} \sigma_{2}\right)^{2}$, and $\rho_{\mathrm{MR}}=A_{\mathrm{MR}} \rho_{2}$.
In order to derive the expressions for the statistical properties of the capacity of double Rice channels, we need the PDF $p_{\Xi^{2}}(z)$ of the squared process $\Xi^{2}(t)$ as well as the joint PDF $p_{\Xi^{2} \dot{\Xi}^{2}}(z, \dot{z})$ of $\Xi^{2}(t)$ and its time derivative $\dot{\Xi}^{2}(t)^{1}$. The joint $p_{\Xi^{2} \dot{\Xi}^{2}}(z, \dot{z})$ can be found using the joint PDF $p_{\Xi \dot{\Xi}}(z, \dot{z}) \quad[10]$ and by using the concept of transformation of random variables [21] as

$$
\begin{aligned}
& p_{\Xi^{2} \dot{\Xi}^{2}}(z, \dot{z})=\frac{1}{4 z} p_{\Xi \dot{\Xi}}\left(\sqrt{z}, \frac{\dot{z}}{2 \sqrt{z}}\right) \\
& =\frac{1}{\sqrt{2 \pi z}\left(4 \pi \sigma_{1} \sigma_{\mathrm{MR}}\right)^{2}} \int_{0}^{\infty} \frac{1}{\sqrt{\beta_{1} y^{4}+\beta_{2} z}} e^{-\frac{z / y^{2}+\rho_{1}^{2}}{2 \sigma_{1}^{2}}} e^{-\frac{y^{2}+\rho_{\mathrm{MR}}^{2}}{2 \sigma_{\mathrm{MR}}^{2}}} \int_{-\pi}^{\pi} e^{-\frac{y \rho_{\mathrm{MR}} \cos \theta_{2}}{\sigma_{\mathrm{MR}}^{2}}} \int_{-\pi}^{\pi} e^{-\frac{1}{2} K^{2\left(\sqrt{z}, y, \theta_{1}, \theta_{2}\right)}} \\
& \times e^{-\frac{(\sqrt{z} / y) \rho_{1} \cos \theta_{1}}{\sigma \frac{2}{1}}} e^{-\frac{(y \dot{z})^{2}}{8 z\left(\beta_{1} y^{4}+\beta_{2} z\right.}-\frac{y \dot{z}}{2 \sqrt{\beta_{1} z y^{4}+\beta_{2} z^{2}}} K\left(\sqrt{Z}, Y, \theta_{1}, \theta_{2}\right)} d \theta_{1} d \theta_{2} d y, \quad z \geq 0,|\dot{z}|<\infty
\end{aligned}
$$

where

$$
\begin{aligned}
& \beta_{1}=2\left(\pi \sigma_{1}\right)^{2}\left(f_{\max _{1}}^{2}+f_{\max _{2}}^{2}\right) \\
& \beta_{2}=2\left(\pi \sigma_{\mathrm{MR}}\right)^{2}\left(f_{\text {max }_{2}}^{2}+f_{\text {max }_{3}}^{2}\right)
\end{aligned}
$$

and

$$
K\left(z, y, \theta_{1}, \theta_{2}\right)=\frac{2 \pi \rho_{1} f_{\rho_{1}} y^{2} \sin \theta_{1}+2 \pi \rho_{\mathrm{MR}} f_{\rho_{2}} z \sin \theta_{2}}{\sqrt{\beta_{1} z y^{4}+\beta_{2} z^{2}}} .
$$

Here $f_{\max _{1}}, f_{\max _{2}}$, and $f_{\max _{3}}$ denote the maximum Doppler frequencies of the SMS, MR and DMS, respectively. The expression for the PDF $p_{\Xi^{2}}(z)$ can be obtained by integrating the joint PDF $p_{\Xi^{2} \dot{\Xi}^{2}}(z, \dot{z})$ over $\dot{z}$. Alternatively, in our case the PDF $p_{\Xi^{2}}(z)$ can also be found from the PDF $p_{\Xi}(z)$ in (3) using the concept of transformation of random variables [21] as

$$
\begin{aligned}
p_{\Xi^{2}}(z)= & \frac{1}{2 \sqrt{z}} p_{\Xi}(\sqrt{z}) \\
= & \frac{1}{2 \sigma_{1}^{2} \sigma_{\mathrm{MR}}^{2}} \int_{0}^{\infty} \frac{1}{y} e^{-\frac{z / y^{2}+\rho_{1}^{2}}{2 \sigma_{1}^{2}}} e^{-\frac{y^{2}+\rho_{\mathrm{MR}}^{2}}{2 \sigma_{\mathrm{MR}}^{2}}} \\
& I_{0}\left(\frac{\sqrt{z} \rho_{1}}{y \sigma_{1}^{2}}\right) I_{0}\left(\frac{y \rho_{\mathrm{MR}}}{\sigma_{\mathrm{MR}}^{2}}\right) d y, \quad z \geq 0 .
\end{aligned}
$$

In the next section, we will derive the statistical properties of the capacity of double Rice channels using the results found in this section.

${ }^{1}$ Throughout this paper, we will represent the time derivative of a process by an overdot.

\section{Statistical Properties of the Capacity of Double Rice Channels}

The instantaneous capacity $C(t)$ of double Rice channels can be expressed using a similar formula found in [22] as

$$
\begin{aligned}
& C(t)=\log _{2}\left(1+\gamma_{s}|\Xi(t)|^{2}\right) \\
& =\log _{2}\left(1+\gamma_{s} \Xi^{2}(t)\right)(\mathrm{bits} / \mathrm{s} / \mathrm{Hz})
\end{aligned}
$$

where $\gamma_{s}$ denotes the average received signal-to-noise ratio (SNR) at the DMS. Equation (9) can be considered as a mapping of a random process $\Xi(t)$ to another random process $C(t)$. Hence, the expressions for the statistical properties of the channel capacity $C(t)$ can be derived by using the results for the statistical properties of the process $\Xi(t)$ obtained in the previous section. The PDF $p_{C}(r)$ of the channel capacity $C(t)$ can be found using (8), (9), and by applying the concept of transformation of random variables as

$$
\begin{aligned}
& p_{C}(r)=\frac{2^{r} \ln (2)}{\gamma_{s}} p_{\Xi^{2}}\left(\frac{2^{r}-1}{\gamma_{s}}\right) \\
&= \frac{2^{r} \ln (2)}{2 \gamma_{s} \sigma_{1}^{2} \sigma_{\mathrm{MR}}^{2}} \int_{0}^{\infty} \frac{1}{y} e^{-\frac{2^{r}-1 / \gamma_{s} y^{2}+\rho_{1}^{2}}{2 \sigma_{1}^{2}}} e^{-\frac{y^{2}+\rho_{\mathrm{MR}}^{2}}{2 \sigma_{\mathrm{MR}}^{2}}} \\
& I_{0}\left(\frac{\sqrt{2^{r}-1} \rho_{1}}{y \sqrt{\gamma_{s}} \sigma_{1}^{2}}\right) I_{0}\left(\frac{y \rho_{\mathrm{MR}}}{\sigma_{\mathrm{MR}}^{2}}\right) d y, r \geq 0 .
\end{aligned}
$$


The $\mathrm{CDF} F_{C}(r)$ of the channel capacity $C(t)$ can now be obtained by solving the integral given by

$$
F_{C}(r)=\int_{0}^{r} p_{C}(z) d z
$$

By substituting (10) in (11) and doing some mathematical manipulations, the CDF of the channel capacity can be expressed as follows

$$
\begin{aligned}
& F_{C}(r)=1-\frac{1}{\sigma_{\mathrm{MR}}^{2}} \int_{0}^{\infty} y e^{-\frac{y^{2}+\rho_{\mathrm{MR}}^{2}}{2 \sigma_{\mathrm{MR}}^{2}}} \\
& I_{0}\left(\frac{y \rho_{\mathrm{MR}}}{\sigma_{\mathrm{MR}}^{2}}\right) Q_{1}\left(\frac{\rho_{1}}{\sigma_{1}}, \frac{\sqrt{2^{r}-1}}{y \sqrt{\gamma_{s}} \sigma_{1}}\right) d y, \quad r \geq 0 \\
& p_{C \dot{C}}(z, \dot{z})=\left(\frac{2^{z} \ln (2)}{\gamma_{s}}\right)^{2} p_{\Xi^{2} \dot{\Xi}^{2}}\left(\frac{2^{z}-1}{\gamma_{s}}, \frac{2^{z} \dot{z} \ln (2)}{\gamma_{s}}\right) \\
& =\frac{\left(2^{z} \ln (2)\right)^{2}\left(2^{z}-1\right)^{-1 / 2}}{(4 \pi)^{2} \sqrt{2 \pi} \gamma_{s}^{3 / 2} \sigma_{1}^{2} \sigma_{\mathrm{MR}}^{2}} \int_{0}^{\infty} \frac{e^{-\frac{2^{z}-1 / \gamma_{s} y^{2}+\rho_{1}^{2}}{2 \sigma_{1}^{2}}}}{\sqrt{\beta_{1} y^{4}+\beta_{2}\left(2^{z}-1 / \gamma_{s}\right)}} e^{-\frac{y^{2}+\rho_{\mathrm{MR}}^{2}}{2 \sigma_{\mathrm{MR}}^{2}}} \int_{-\pi}^{\pi} e^{-\frac{y \rho_{\mathrm{MR}} \cos \theta_{2}}{\sigma_{\mathrm{MR}}^{2}}} \int_{-\pi}^{\pi} e^{-\frac{\sqrt{2^{z}-1 / \gamma_{s} y^{2}} \rho_{1} \cos \theta_{1}}{\sigma_{1}^{2}}} \\
& \times e^{-\frac{\left(y \dot{z} \ln (2) 2^{z}\right)^{2}\left(2^{z}-1\right)^{-1}}{8\left(\gamma_{s} \beta_{1} y^{4}+\beta_{2}\left(2^{z}-1\right)\right)}} e^{-\frac{y \dot{z} \ln (2) 2^{z}\left(2^{z}-1\right)^{-1 / 2}}{2 \sqrt{\gamma_{s} \beta_{1} y^{4}+\beta_{2}\left(2^{z}-1\right)}} K\left(\sqrt{\frac{2^{z}-1}{\gamma_{s}}, y, \theta_{1}, \theta_{2}}\right)} e^{-\frac{1}{2} K^{2}\left(\sqrt{\left(2^{z}-1 / \gamma_{s}\right)}, y, \theta_{1}, \theta_{2}\right)} d \theta_{1} d \theta_{2} d y
\end{aligned}
$$

for $z \geq 0,|\dot{z}|<\infty$, where $K(,,,,$,$) is defined in (7). After substituting (14) in (13) and carrying out some algebraic cal-$ culations, we obtain

$$
\begin{aligned}
& N_{C}(r)=\frac{\sqrt{2^{r}-1}}{(2 \pi)^{5 / 2} \sqrt{\gamma_{s}} \sigma_{1}^{2} \sigma_{\mathrm{MR}}^{2}} \int_{0}^{\infty} \sqrt{\beta_{1}+\beta_{2}\left(\frac{2^{r}-1}{y^{4} \gamma_{s}}\right)} e^{-\frac{2^{r}-1 / \gamma_{s} y^{2}+\rho_{1}^{2}}{2 \sigma_{1}^{2}}} e^{-\frac{y \rho_{\mathrm{MR}} \cos \theta_{2}}{\sigma_{\mathrm{MR}}^{2}}} \int_{-\pi}^{\pi} e^{-\frac{\sqrt{2^{r}-1 / \gamma_{s} y^{2}} \rho_{1} \cos \theta_{1}}{\sigma_{1}^{2}}} \\
& \times \int_{-\pi}^{\pi}\left[1+\sqrt{\frac{\pi}{2}} e^{\frac{1}{2} K^{2}\left(\sqrt{\left(2^{r}-1 / \gamma_{s}\right)}, y, \theta_{1}, \theta_{2}\right)} \quad K\left(\sqrt{\frac{2^{r}-1}{\gamma_{s}}}, y, \theta_{1}, \theta_{2}\right) \quad\left\{1+\Phi\left(\frac{K\left(\sqrt{\frac{2^{r}-1}{\gamma_{s}}, y, \theta_{1}, \theta_{2}}\right)}{\sqrt{2}}\right)\right\}\right] \\
& \times e^{-\frac{y^{2}+\rho_{\mathrm{MR}}^{2}}{2 \sigma_{\mathrm{MR}}^{2}}} e^{-\frac{1}{2} K^{2}\left(\sqrt{\left(2^{r}-1 / \gamma_{s}\right)}, y, \theta_{1}, \theta_{2}\right)} d \theta_{1} d \theta_{2} d y, r \geq 0
\end{aligned}
$$

where $\Phi(\cdot)$ denotes the error function [20]. Finally, from (15) and (12), the $\mathrm{ADF} T_{C}(r)$ of the channel capacity $C(t)$ can be obtained using [19]

$$
T_{C}(r)=\frac{F_{C}(r)}{N_{C}(r)} .
$$

The results found in this section will be used in the following section to derive the statistical properties of the capacity of double Rayleigh channels.

\section{Statistical Properties of the Capacity of Double Rayleigh Channels}

The double Rayleigh channel follows as a special case of 
the double Rice channel when $\rho_{i} \rightarrow 0 \quad(i=1,2)$. Hence, by letting $\rho_{i} \rightarrow 0 \quad(i=1,2)$ in (10), (12), and (15), the PDF, CDF, and LCR of the channel capacity of double Rayleigh channel can be expressed as

$$
\begin{gathered}
\left.p_{C}(r)\right|_{\rho_{i} \rightarrow 0}=\frac{2^{r} \ln (2)}{2 \gamma_{s} \sigma_{1}^{2} \sigma_{\mathrm{MR}}^{2}} \int_{0}^{\infty} \frac{1}{y} e^{-\frac{2^{r}-1}{2 \sigma_{1}^{2} \gamma_{s} y^{2}}} e^{-\frac{y^{2}}{2 \sigma_{\mathrm{MR}}^{2}}} d y, r \geq 0 \\
\left.F_{C}(r)\right|_{\rho_{i} \rightarrow 0}=1-\frac{1}{\sigma_{\mathrm{MR}}^{2}} \int_{0}^{\infty} y e^{-\frac{y^{2}}{2 \sigma_{\mathrm{MR}}^{2}}} e^{-\frac{2^{r}-1}{2 \sigma_{1}^{2} \gamma_{s} y^{2}}} d y, r \geq 0
\end{gathered}
$$

and

$$
\begin{aligned}
\left.N_{C}(r)\right|_{\rho_{i} \rightarrow 0}= & \frac{\sqrt{2^{r}-1}}{\sqrt{2 \pi \gamma_{s}} \sigma_{1}^{2} \sigma_{\mathrm{MR}}^{2}} \\
& \int_{0}^{\infty} \sqrt{\beta_{1}+\beta_{2}\left(\frac{2^{r}-1}{y^{4} \gamma_{s}}\right)} e^{-\frac{2^{r}-1}{2 \sigma_{1}^{2} \gamma_{s} y^{2}}} e^{-\frac{y^{2}}{2 \sigma_{\mathrm{MR}}^{2}}} d y, r \geq 0
\end{aligned}
$$

respectively. The ADF of the channel capacity $C(t)$ of double Rayleigh channel can be found using (16), (18), and (19).

\section{Statistical Properties of the Capacity of Rayleigh and Rice Channels}

In this section, we will present the PDF, CDF, and LCR of the capacity of Rayleigh and Rice channels. We will study these results along with the statistical properties of the capacity of double Rice channels in the next section for comparison purposes. The PDF $p_{C}(r)$ of the capacity $C(t)$ of Rice channels can be found using the PDF $p_{\xi^{2}}(r)$ of the squared Rice process $\xi^{2}(t)$ and by employing the expression presented in (9) corresponding to Rice processes $\xi(t)$ as

$$
\begin{aligned}
p_{C}(r) & =\frac{2^{r} \ln (2)}{\gamma_{s}} p_{\xi^{2}}\left(\frac{2^{r}-1}{\gamma_{s}}\right) \\
& =\frac{2^{r} \ln (2)}{2 \gamma_{s} \sigma_{0}^{2}} e^{-\frac{2^{r}-1+\gamma_{s} \rho^{2}}{2 \sigma_{0}^{2} \gamma_{s}}} I_{0}\left(\sqrt{\frac{\left(2^{r}-1\right) \rho^{2}}{\sigma_{0}^{4} \gamma_{s}}}\right), \quad r \geq 0
\end{aligned}
$$

where $\rho$ represent the amplitude of the LOS component and $\sigma_{0}^{2}$ denotes the variance of the underlying Gaussian processes. By substituting (20) in $F_{C}(r)=\int_{0}^{r} p_{C}(x) d x$, the CDF $F_{C}(r)$ of the capacity of $C(t)$ of Rice channels can be written as

$$
F_{C}(r)=1-Q_{1}\left(\frac{\rho}{\sigma_{0}}, \sqrt{\frac{\left(2^{r}-1\right)}{\sigma_{0}^{2} \gamma_{s}}}\right), \quad r \geq 0
$$

By solving $N_{C}(r)=\int_{0}^{\infty} p_{C \dot{C}}(r, \dot{z}) d \dot{z}$, the LCR $N_{C}(r)$ of the capacity of $C(t)$ of Rice channels can be represented by

$$
N_{C}(r)=\sqrt{\frac{\left(2^{r}-1\right) \beta}{\pi 2 \sigma_{0}^{4} \gamma_{s}}} e^{-\frac{2^{r}-1+\gamma_{s} \rho^{2}}{2 \sigma_{0}^{2} \gamma_{s}}} I_{0}\left(\sqrt{\frac{\left(2^{r}-1\right)}{\sigma_{0}^{4} \gamma_{s} / \rho^{2}}}\right), \quad r \geq 0
$$

where $\beta$ under isotropic scattering conditions is given by $\beta=2\left(\pi f_{\max } \sigma_{0}\right)^{2}$. Here, $p_{C \dot{C}}(z, \dot{z})$ represents the joint PDF of the capacity $C(t)$ and its time derivative $\dot{C}(t)$ and $f_{\max }$ denotes the maximum Doppler frequency.

The results for the PDF, CDF, and LCR of the capacity $C(t)$ of Rayleigh channels can be obtained from (20)-(22), respectively, by letting $\rho \rightarrow 0$ as follows:

$$
\begin{aligned}
& \left.p_{C}(r)\right|_{\rho \rightarrow 0}=\frac{2^{r} \ln (2)}{2 \gamma_{s} \sigma_{0}^{2}} e^{-\left(\frac{2^{r}-1}{2 \gamma_{s} \sigma_{0}^{2}}\right)}, r \geq 0 \\
& \left.F_{C}(r)\right|_{\rho \rightarrow 0}=1-e^{-\left(\frac{2^{r}-1}{2 \gamma_{s} \sigma_{0}^{2}}\right)}, r \geq 0 \\
& \left.N_{C}(r)\right|_{\rho \rightarrow 0}=\frac{1}{\sigma_{0}^{2}} \sqrt{\frac{\beta\left(2^{r}-1\right)}{2 \pi \gamma_{s}}} e^{-\left(\frac{2^{r}-1}{2 \gamma_{s} \sigma_{0}^{2}}\right)}, r \geq 0 .
\end{aligned}
$$

The expressions (23)-(25) have already been presented in [23, Equations (23-25)]. However, we have presented these equations here for the sake of completeness.

\section{Numerical Results}

This section aims at the validation and analysis of the analytical results presented in the previous section, using simulations. We have also included the results for double Rayleigh, classical Rayleigh [19], and classical Rice channels in our study for comparison purposes. For the case of classical Rice channels, we denote the amplitude of the LOS component as $\rho$. The Rice processes $\mu_{\rho}^{(i)}(t)=\mu^{(i)}(t)+m^{(i)}(t) \quad(i=1,2) \quad$ were simulated using the sum-of-sinusoids model [24]. The model parameters were computed using the generalized method of exact Doppler spread $\left(\mathrm{GMEDS}_{1}\right)$ [25]. The number of sinusoids $\left(N_{1}^{(i)}\right.$ and $\left.N_{2}^{(i)}\right)$ for the resulting deterministic processes $\tilde{\mu}_{1}^{(i)}(t)$ and $\widetilde{\mu}_{2}^{(i)}(t)$ in $\operatorname{GMEDS}_{1}$ were chosen to be $N_{1}^{(i)}=N_{2}^{(i)}=20$ for $i=1,2$, respectively. The maximum Doppler frequencies $f_{\max _{2}}$ and $f_{\max _{3}}$ were taken to be 91 and $125 \mathrm{~Hz}$, respectively. We have assumed that the Doppler frequency $f_{\rho_{\mathrm{SMS}}}$ equals 0 . Unless stated otherwise, the values of the Doppler fre- 
quencies $f_{\rho_{\mathrm{MR}}}$ and $f_{\rho_{\mathrm{DMS}}}$ were set to be equal to $f_{\max _{2}}$ and $f_{\max _{3}}$, respectively. The SNR $\gamma_{s}$ was set to $20 \mathrm{~dB}$. The parameters $A_{\mathrm{MR}}$ and $\sigma_{i} \quad(i=1,2)$ were chosen to be unity. Finally, using (2) and (9) the simulation results for the statistical properties of the channel capacity were found.

The PDF $p_{\Xi}(z)$ of double Rice processes $\Xi(t)$ are shown in Figure 2 for different values of the amplitudes of the LOS components $\rho_{i} \quad(i=1,2)$. In Figure 2, the PDF of the double Rayleigh process is also shown, which represents a special case of the double Rice process when $\rho_{1}=\rho_{2}=0$. It is observed that the presence of the LOS components has a dominant effect on the mean value and spread of the PDF of double Rice processes. It can also be seen that the PDF of double Rice processes is identical for the cases $\rho_{2}=0 ; \rho_{1}=2$ and $\rho_{2}=2 ; \rho_{1}=0$.

Figures 3 and 4 present the PDF and CDF of the capacity of double Rice channels, respectively. It is observed that the amplitude of the LOS component has a significant influence on the PDF and CDF of the channel capacity. Specifically, the presence of an LOS component in one or both of the links (i.e., the SMS-MR and MR-DMS links) increases the mean channel capacity. Hence, double Rayleigh channels have a lower mean channel capacity compared to double Rice channels, (e.g., when $\left.\rho_{2}=\rho_{1}=2\right)$. It is also observed that the capacity of classical Rice channels has a lower mean value compared to that of double Rice channels. These facts are specifically studied in Figure 5, where the mean channel capacity of classical Rice as well as double Rice channels is studied for different values of the amplitudes of the LOS component. Figure 6 shows the influence of the amplitude of LOS component on the variance of the classical Rice and double Rice channels. It is observed that increasing the value of $\rho$ decreases the spread of the channel capacity for medium and large values of $\rho$, say $\rho \geq 1$. Moreover, the variance of the capacity of double Rice channels is much higher as compared to that of classical Rice channels.

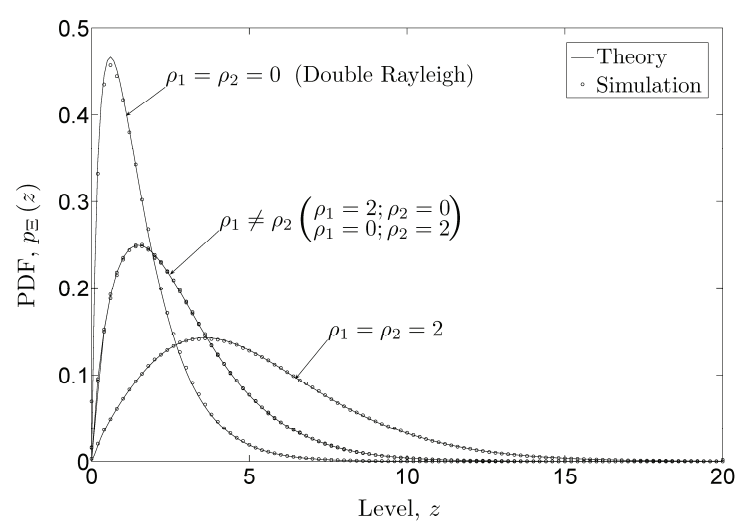

Figure 2. The PDF $p_{\Xi}(z)$ of double Rice processes $\Xi(t)$.
The LCR and ADF of the channel capacity of double Rice channels are presented in Figures 7 and 8, respectively. It is evident from Figure 7 that the maximum value of the LCR of the channel capacity increases with an increase in the value of the amplitude of the LOS component $\rho_{i} \quad(i=1,2)$. It is also observed that the LCR of the capacity of classical Rice channels is much lower compared to that of double Rice channels. The converse statements with respect to the LCR of the channel capacity are true for the ADF, as can be seen in Figure 8 .

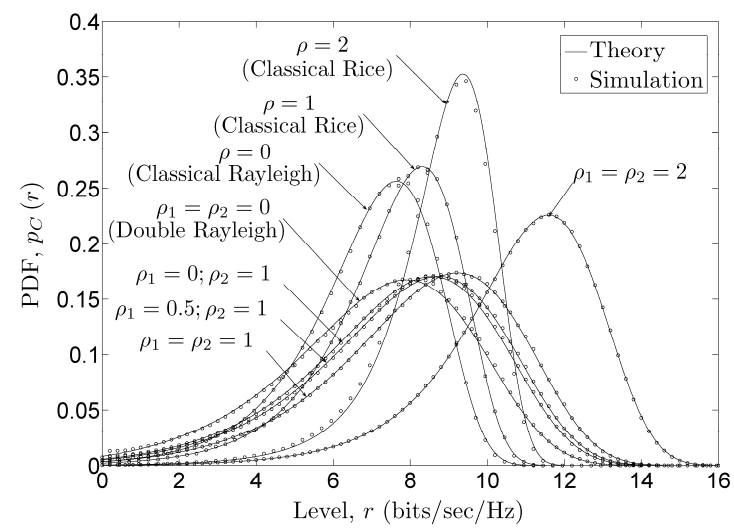

Figure 3. The PDF $p_{C}(r)$ of the capacity of double Rice channels.

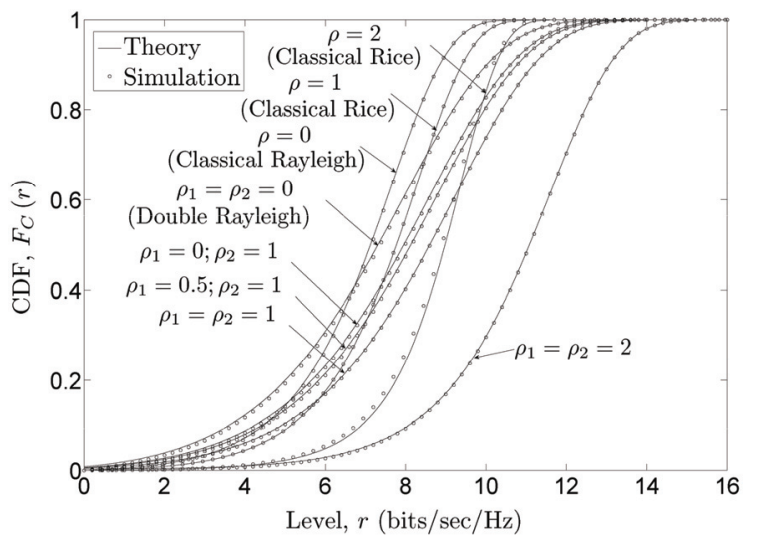

Figure 4. The CDF $F_{C}(r)$ of the capacity of double Rice channels.

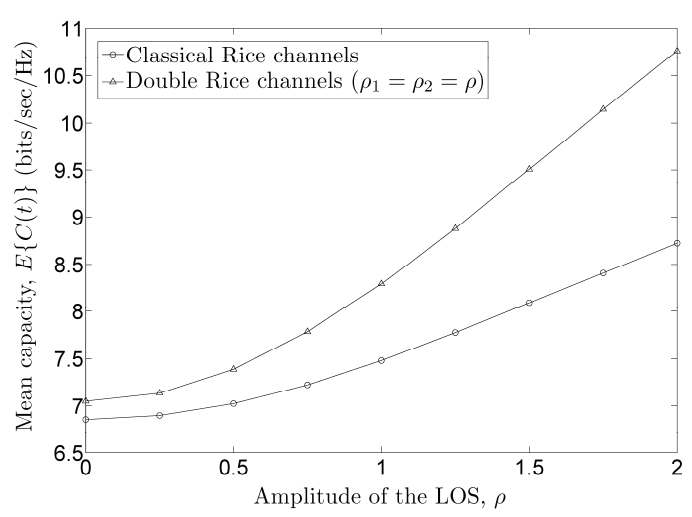

Figure 5. The mean capacity $E\{C(t)\}$ of classical Rice and double Rice channels. 


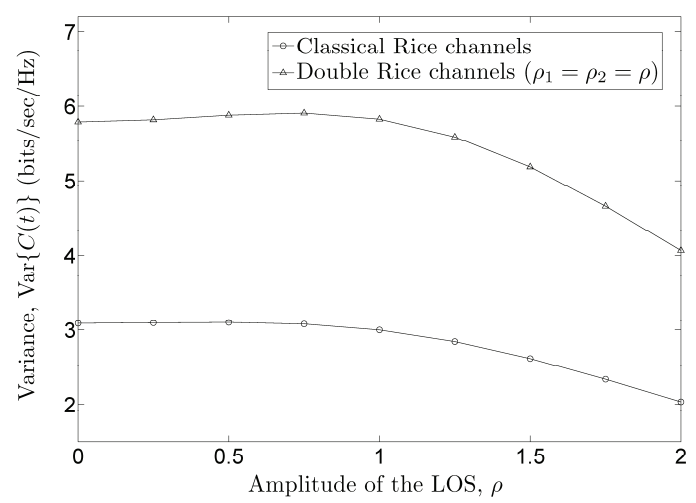

Figure 6. The variance $\operatorname{Var}\{C(t)\}$ of the capacity of classical Rice and double Rice channels.

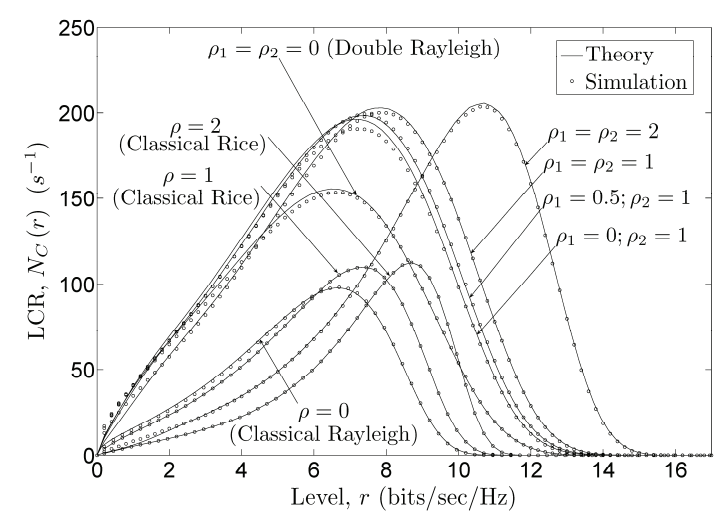

Figure 7. The LCR $N_{C}(r)$ of the capacity of double Rice channels.

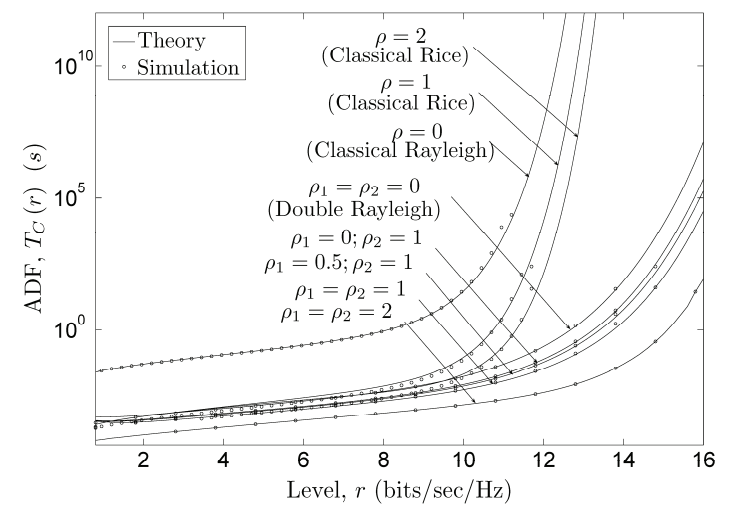

Figure 8. The ADF $T_{C}(r)$ of the capacity of double Rice channels.

Figures 9 and 10 illustrate the effect of the Doppler frequency on the LCR and ADF of the channel capacity. From Figures 9 and 10, it can clearly be seen that the LCR and ADF are strongly dependent on the Doppler frequencies of the MR and the DMS. It is observed that increasing the Doppler frequencies $f_{\rho_{\mathrm{MR}}}$ and $f_{\rho_{\mathrm{DMS}}}$ from 0 to $f_{\max _{2}}$ and $f_{\max _{3}}$, respectively, results in a significant increase in the LCR. However, the ADF decreases by increasing the Doppler frequencies of the MR and the DMS.

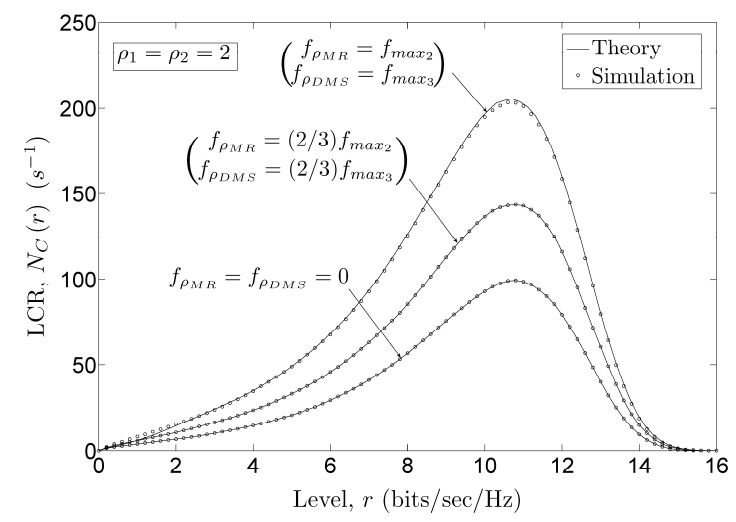

Figure 9. The LCR $N_{C}(r)$ of the capacity of double Rice channels.

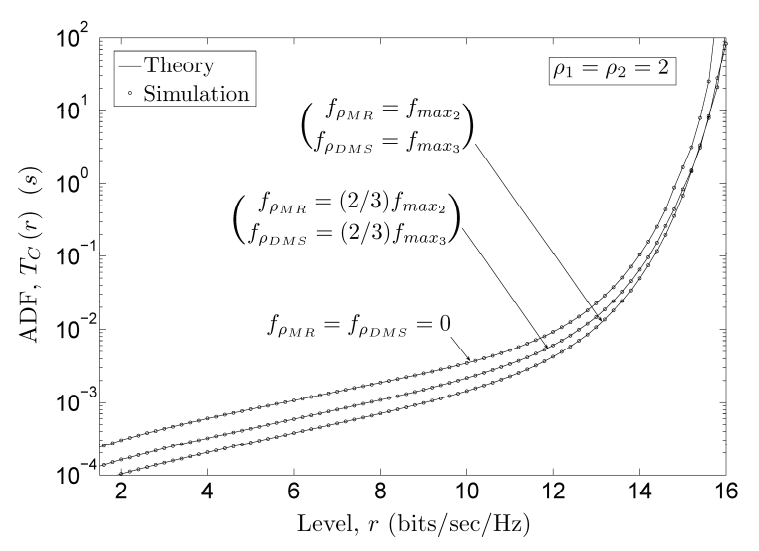

Figure 10. The ACF $T_{C}(r)$ of the capacity of double Rice channels.

\section{Conclusions}

In this paper, we have studied the statistical properties of the channel capacity of the double Rice channels. We have derived analytical expressions for the PDF, CDF, LCR, and ADF of the channel capacity. The findings of this paper give a deep insight into the influence of the LOS components, corresponding to the two links of amplify-and-forward channels, on the statistical properties of the channel capacity. It has been observed that for medium and high signal levels, the presence of the LOS components in one or both of the links of the double Rice channel model increases the mean channel capacity and the LCR of the channel capacity. However, it decreases the ADF of the channel capacity. Moreover, the Doppler frequencies of the MR and the DMS have a significant impact on the LCR and ADF of the channel capacity. The validity of all the presented analytical results is confirmed by simulations, whereby a very good fitting between the analytical and simulation results is found.

\section{References}

[1] A. Sendonaris, E. Erkip, and B. Aazhang, "User coopera- 
tion diversity-Part I: System description," IEEE Transactions on Communications, Vol. 51, No. 11, pp. 1927-1938, November 2003.

[2] A. Sendonaris, E. Erkip, and B. Aazhang, "User cooperation diversity - Part II: Implementation aspects and performance analysis," IEEE Transactions on Communications, Vol. 51, No. 11, pp. 1939-1948, November 2003.

[3] J. N. Laneman, D. N. C. Tse, and G. W. Wornell, “Cooperative diversity in wireless networks: Efficient protocols and outage behavior," IEEE Transactions on Information Theory, Vol. 50, No. 12, pp. 3062-3080, December 2004.

[4] M. Dohler, "Virtual antenna arrays," Ph. D. dissertation, King's College, London, United Kingdom, 2003.

[5] J. B. Andersen, "Statistical distributions in mobile communications using multiple scattering," in Proceedings of 27th URSI General Assembly, Maastricht, Netherlands, August 2002.

[6] I. Z. Kovacs, P. C. F. Eggers, K. Olesen, and L. G. Petersen, "Investigations of outdoor-to-indoor mobile-to-mobile radio communication channels," in Proceedings of IEEE 56th Vehicular Technology Conference, VTC'02 Fall, Vancouver BC, Canada, pp. 430-434, September 2002.

[7] V. Ercerg, S. J. Fortune, J. Ling, A. J. Rustako Jr., and R. A. Valenzuela, "Comparison of a computer based propagation prediction tool with experimental data collected in urban microcellular environment," IEEE Journal on Selected Areas in Communications, Vol. 15, No. 4, pp. 677-684, May 1997.

[8] C. S. Patel, G. L. Stüber, and T. G. Pratt, "Statistical properties of amplify and forward relay fading channels," IEEE Transactions on Vehicular Technology, Vol. 55, No. 1, pp. 1-9, January 2006.

[9] J. Salo, H. M. El Sallabi, and P. Vainikainen, "Statistical analysis of the multiple scattering radio channel," IEEE Transactions on Antennas Propagation, Vol. 54, No. 11, pp. 3114-3124, November 2006.

[10] B. Talha and M. Pätzold, "On the statistical properties of double Rice channels," in Proceedings of 10th International Symposium on Wireless Personal Multimedia Communications, WPMC 2007, Jaipur, India, pp. 517-522, December 2007.

[11] B. Talha and M. Pätzold, "On the statistical properties of mobile to mobile fading channels in cooperative networks under line of sight conditions," in Proceedings of 10th International Symposium on Wireless Personal Multimedia Communications, WPMC 2007. Jaipur, India, pp. 388-393, December 2007.

[12] B. Talha and M. Pätzold, "A novel amplify-and-forward relay channel model for mobile-to-mobile fading channels under line-of-sight conditions," in Proceedings of 19th IEEE International Symposium on Personal, Indoor and Mobile Radio Communications, PIMRC 2008, Cannes, France, September 2008

[13] B. Talha and M. Pätzold, "Level-crossing rate and average duration of fades of the envelope of mobile- to-mobile fading channels in cooperative networks under line-of-sight conditions," in IEEE Conference Proceedings Globecom'08, New Orleans, LA, USA, November /December 2008.

[14] J. Salo, H. M. El-Sallabi, and P. Vainikainen, "Impact of double-Rayleigh fading on system performance," in Proceedings of 1st IEEE International Symposium on Wireless Pervasive Computing, ISWPC 2006. Phuket, Thailand, January 2006.

[15] P. Almers, F. Tufvesson, and A. F. Molisch, "Keyhole effect in MIMO wireless channels: Measurements and theory," IEEE Transactions on Wireless Communications, Vol. 5, No. 12, pp. 3596-3604, 2006.

[16] D. Gesbert, H. Bölcskei, D. A. Gore, and A. J. Paulraj, "Outdoor MIMO wireless channels: Models and performance prediction," IEEE Transactions on Wireless Communications, Vol. 50, No. 12, pp. 1926-1934, December 2002.

[17] A. Giorgetti, P. J. Smith, M. Shafi, and M. Chiani, "MIMO capacity, level crossing rates and fades: The impact of spatial/temporal channel correlation," Journal of Communications and Networks, Vol. 5, No. 2, pp. 104-115, June 2003.

[18] B. O. Hogstad and M. Pätzold, "Capacity studies of MIMO models based on the geometrical one-ring scattering model," in Proceedings of 15th IEEE International Symposium on Personal, Indoor and Mobile Radio Communications, PIMRC 2004, Vol. 3. Barcelona, Spain, pp. 1613-1617, September 2004.

[19] B. O. Hogstad and M. Pätzold, "Exact closed-form expressions for the distribution, level- crossing rate, and average duration of fades of the capacity of MIMO channels," in Proceedings of 65th Semiannual Vehicular Technology Conference, IEEE VTC 2007-Spring, Dublin, Ireland, pp. 455-460, April 2007.

[20] I. S. Gradshteyn and I. M. Ryzhik, "Table of Integrals, Series, and Products," 6th edition, Academic Press, 2000.

[21] A. Papoulis and S. U. Pillai, Probability, "Random variables and stochastic processes," 4th edition, New York: McGraw-Hill, 2002.

[22] G. J. Foschini and M. J. Gans, "On limits of wireless communications in a fading environment when using multiple antennas," Wireless Personal Communications, Vol. 6, pp. 311-335, March 1998.

[23] G. Rafiq and M. Pätzold, "A study of the influence of shadowing on the statistical properties of the capacity of mobile radio channels," Wireless Personal Communications, DOI 10.1007/s11277-008-9545-7, June 2008.

[24] M. Pätzold, "Mobile fading channels," Chichester: John Wiley \& Sons, 2002.

[25] M. Pätzold and B. O. Hogstad, "Two new methods for the generation of multiple uncorrelated Rayleigh fading waveforms," in Proceedings of 63rd IEEE Semiannual Vehicular Technology Conference, IEEE VTC 2006-Spring, Vol. 6, Melbourne, Australia, pp. 2782-2786, May 2006. 